\title{
C. PROPER MOTIONS AND RADIAL VELOGITIES
}

One of the most important fields for co-ordination in galactic research is the organization of programmes for proper motions and radial velocities. The need for accurate and more extensive data on stellar motions is strongly felt in connexion with the studies of the structure of the Galaxy and of the relation between the physical and the kinematical characteristics of the stars. Not long ago it seemed that meridian astronomy and photographic astrometry were rather remote from the main problems in astrophysics. It is realized now, that the study of stellar motions gives highly important information on the evolution of the stars and of the stellar system. A detailed account of the needs in this field, as discussed at the conference, follows:

\section{Variable stars}

\section{(I) PROPER MOTIONS: PHOTOGRAPHIC ASTROMETRY OF FAINT STARS}

Among the variable stars we find the most pronounced types of halo stars and typical disk population II. They are the main source of information on the motions of population II objects. They are scarce objects in the neighbourhood of the Sun, and their study, therefore, requires observations up to $m_{p g}=\mathrm{I} 2$ or fainter.

Current programmes at various observatories are:

The determination of proper motions of RR Lyrae stars at the Mount Wilson Observatory, in collaboration with the Leiden Observatory, and at the Leander McCormick Observatory.

The determination of proper motions of long-period variables at the Leander McCormick Observatory and at the Leiden Observatory.

The determination of proper motions of faint variable stars by different astronomers at the Sternberg State Astronomical Institute, Moscow, by comparison of plates taken with the $3^{8} \mathrm{~cm}$. astrograph with first epoch Astrographic Catalogue plates, or with first epoch plates taken at Moscow since 1932. (New results for $3^{6} \mathrm{RR}$ Lyrae stars have been published recently by Pavlov. ${ }^{1}$ )

Although these programmes will soon considerably increase our knowledge of the motions of these objects, any attempt at further work on them 
should be encouraged. It is a favourable circumstance that some of the observatories which took part in the early work on the Astrographic Catalogue have indicated a desire to use the old epoch plates for proper motion work. Therefore, a list has been prepared by Dr L. Plaut of the Kapteyn Laboratory, of variable stars brighter than photographic or photovisual magnitude 12.0 in maximum, occurring in Parenago and Kukarkin's catalogue. The list contains 2000 RR Lyrae, long-period, semi-regular and RV Tauri variables, and these are arranged in the order of the Astrographic Catalogue zone in which they occur. $\delta$ Cephei variables are not included; because of the smallness of their proper motions, these can be studied only with meridian instruments. The list will be made available to all institutions interested in this work, through the intermediary of the Commission for the Astrographic Catalogue, and may be published in the next report of that Commission. Preliminary work in this field shows that about half of the stars in this list do indeed occur in the Astrographic Catalogue. Among the missing ones, many can be measured on Astrographic Chart plates when these are available.

This list may be followed by others when the need arises for measurement of other types of stars of special interest.

For almost all purposes for which proper motions are studied, it is necessary that the relative proper motions are reduced to an absolute system. This is always necessary, for instance, when proper motions are converted into linear space velocities. One way of doing this, is to assume a mean proper motion of the reference stars by adopting the most probable value for the parallactic motion and for the differential galactic rotation. A more satisfactory procedure is that of reducing the motions to a fundamental system. (See also Vyssotsky's discussion in the Reports on the Dearborn Astrometric Conference, A.F. 59, No. 2.) Reduction to a fundamental system will be possible for stars of the magnitude range measured on the Astrographic Catalogue plates (photographic magnitudes 9-12) when a repetition of the $\mathrm{AGK}_{2}$ (see Section $\mathrm{C}(3)$ ) has been carried out.

In view of these considerations Parenago proposed that, together with the results for the stars of the particular programme, the reference stars, their apparent magnitudes and their measured relative proper motions are also published. One might even consider measuring the AG stars present on the plates in order to make sure that reduction to an absolute system can be accomplished as soon as the $\mathrm{AGK}_{2}$ repetition is completed. 
It must also be emphasized here, that in those cases where the reduction to absolute has to be based on the parallactic motion of the reference stars, at least twenty reference stars are needed to reduce the influence of their peculiar motions to a satisfactory minimum.

\section{Kapteyn's Selected Areas}

Proper motions in the northern Selected Areas have been published by the Pulkovo and Radcliffe Observatories. ${ }^{1}$ The southern areas are now being measured at the Kapteyn Laboratory on plates taken at the southern station of the Yale Observatory.

Repetition of these Radcliffe and Pulkovo observations in the northern Selected Areas could give a ten-fold increase of the weight of the proper motions, and probable errors of the order of o"oor. Such very accurate data would allow a much more refined study of the velocity distribution of distant objects than was hitherto possible. The great amount of work involved might make it desirable to confine the repetition to part of the areas. In that case, preference should be given to the low galactic latitudes (for instance, those below $20^{\circ}$ ) where the proper motions are smallest and where the motions are most complicated. These low latitude areas cover $4 \mathrm{o}^{\prime} \times 4 \mathrm{o}^{\prime}$ and the limiting photographic magnitude is about 14.5 .

Even if such a programme is undertaken, there will remain a strong nẹed for proper motions and radial velocities of many more low latitude stars brighter than 12.5 . This is the limiting magnitude up to which we eventually may expect to get accurate spectral classifications and radial velocities. It is also the limiting magnitude of the Astrographic Catalogue. Therefore it was stressed, particularly by Oort and Van Rhijn, that a very important task for the observatories equipped with Astrographic Catalogue refractors will be the determination of proper motions in regions centred on the Kapteyn Selected Areas, but much larger than those given in the Pulkovo and Radcliffe Catalogues. The choice of the Kapteyn Areas is obvious, because here we have already magnitudes, colours, and spectral classifications available. The size of the areas should be taken $3.5^{\circ} \times 3.5^{\circ}$, as in the Bergedorfer Spectral Durchmusterung. (The Potsdam Spectral Durchmusterung, which covers the southern Kapteyn Areas, has even larger fields and reaches stars of about 12.0 and brighter.)

An average low latitude area of $3.5^{\circ} \times 3.5^{\circ}$ contains, between photographic magnitude 11.5 and 12.5 , about 150 A stars, $250 \mathrm{G}$ stars and $60 \mathrm{~K}$ stars. With the subdivisions into giants and dwarfs and possibly also into strong- and weak-line stars, there remain only relatively small numbers of stars per area for statistical investigation. It is, therefore, proposed 
that the project includes all Kapteyn Selected Areas below $10^{\circ}$ galactic latitude. A list of these areas and their co-ordinates follows.

Selected Areas below $10^{\circ}$ galactic latitude

\begin{tabular}{|c|c|c|c|c|c|c|c|c|c|c|}
\hline No. & $\underset{(\mathbf{I} 9}{\mathbf{R}}$ & $\begin{array}{l}\text { A. } \\
\text { oo) }\end{array}$ & $\begin{array}{c}\text { Decl. } \\
\text { (19oo) }\end{array}$ & $l$ & $b$ & No. & $\begin{array}{c}\text { R.A. } \\
\text { (1900) }\end{array}$ & $\begin{array}{l}\text { Decl. } \\
(\mathrm{r} 900)\end{array}$ & $l$ & $b$ \\
\hline 8 & $I^{h}$ & $\mathbf{o}^{\mathbf{m}}$ & $+60^{\circ} 10^{\prime}$ & $92^{\circ}$ & $-2^{\circ}$ & IIO & $18^{\mathrm{h}} 37^{\mathrm{m}}$ & $0^{\circ} \mathrm{oo}^{\prime}$ & $0^{\circ}$ & $1^{\circ}$ \\
\hline 9 & & 4 & $\begin{array}{ll}60 & 20\end{array}$ & Io6 & 3 & 123 & 765 & -1500 & 197 & o \\
\hline 18 & & 24 & $60 \quad 10$ & 68 & 6 & I 34 & 1810 & -1500 & 343 & $-I$ \\
\hline 19 & 23 & 23 & 6000 & $8 \mathrm{r}$ & $-I$ & 147 & 73 & -3010 & 209 & -9 \\
\hline 23 & 3 & 39 & 4500 & 120 & -7 & 148 & $75^{8}$ & -30 10 & 215 & I \\
\hline 24 & 4 & 39 & $445^{\circ}$ & 128 & 0 & 157 & $17 \quad 25$ & -30 10 & 325 & I \\
\hline 25 & 5 & 37 & 4450 & I33 & 9 & 172 & 840 & -45 го & 232 & -1 \\
\hline 39 & 19 & 47 & $445^{\circ}$ & 47 & 9 & I 73 & 935 & -45 I0 & 239 & 6 \\
\hline 40 & 20 & 47 & 4500 & 53 & 0 & I 79 & 1548 & -45 го & 301 & 5 \\
\hline $4^{I}$ & 21 & 50 & 4500 & 6I & -8 & 180 & I6 46 & -45 oo & 308 & -2 \\
\hline 49 & 5 & 24 & 2940 & I 45 & -2 & 192 & 926 & -60 oo & 248 & -7 \\
\hline 64 & 19 & $5^{8}$ & 3000 & 35 & $-I$ & 193 & I1 27 & -60 Io & $26 I$ & I \\
\hline 74 & 6 & 15 & 1510 & 163 & I & I 94 & 1257 & -60 10 & 272 & 2 \\
\hline 87 & 19 & II & 1500 & 17 & o & 195 & 1457 & -60 10 & 286 & -3 \\
\hline $9^{8}$ & 6 & 47 & - 0 I0 & 18I & $\mathbf{I}$ & & & & & \\
\hline
\end{tabular}

In addition, this programme of proper motions and radial velocities should include the high latitude areas mentioned in Section B (2), which will be selected by the continuation committee.

Future matters for organization will be the extension to some other particularly transparent low latitude regions and to some areas at intermediate latitude.

Another suggestion for proper motion measurements are the remaining regions in which Fehrenbach has measured objective prism radial velocities (see p. 4I).

\section{Milky Way regions of special interest}

Proper motions in the low latitude fields, in which luminosity classifications have been made at Gleveland, would be of great value for the statistical study of these data. (See reference no. I for page 29.) The same holds for the test areas mentioned in Section B (I).

\section{T Tauri stars}

Surveys of $\mathrm{T}$ Tauri stars are now being carried out at the Tonantzintla and Lick Observatories (Haro, Herbig). Work on proper motions of the nearest objects (Parenago, Pels and Uranova) indicates that their motions are fairly large $( \pm 12 \mathrm{~km}$. $/ \mathrm{sec}$.) and more precise measurements especially of the nearer groups of these stars within 500 parsecs, may prove very valuable in the interpretation of these objects. (See also p. 44.) 
Stars in the outer regions of open clusters

For most clusters, only the central part has been thoroughly studied. Measurements in the outer parts will be important in order to determine how far from the centre the cluster extends. A survey of the Hyades in a region $5 \times 5$ degrees up to photographic magnitudes 14.5 and in a region Io $\times$ Io degrees around Praesepe, $m<10$, is reported by Heckmann. A search for fainter members $(m<12)$ in a large area around the Hyades (about $30 \times 30$ degrees) is being carried out by Pels at the Leiden Observatory. It is based on a comparison of Astrographic Catalogue positions with modern ones. Work done at Hamburg indicates expansion in the Eddington Perseus cluster. It would be desirable to study this also in other clusters.

\section{Late M-type giants found in the infra-red survey at Cleveland}

Proper motions of these stars will help in the determination of their absolute magnitudes, about which there is considerable uncertainty at present. A list of $\mathrm{I} 20$ of the brighter objects (photographic magnitudes $<$ I3) has been made available by Dr Nassau and will be sent to institutions interested in this work.

\section{Stars with large proper motion}

. The study of the faint stars with large proper motion is important for the determination of the faint end of the luminosity law and also because among these we encounter dwarf stars with high space velocities. Some of these have come from the inner regions of the Galaxy and are, therefore, particularly interesting for further spectroscopic investigation.

For both these problems we require a more accurate determination of the proper motion than is available at present. A programme of such measures could very well be carried out with the aid of the Astrographic Catalogue or chart plates. The stars to be measured could be picked out principally from the lists by Luyten, Ross and Wolf.

Luyten's Bruce Proper Motion Survey covers the southern hemisphere. A list of the stars with large proper motions found in this survey (most of them exceeding $0^{\prime \prime} 05$ ) for declinations below $-50^{\circ}$ has been published in the General Catalogue of the Bruce Proper Motion Survey. It covers all stars up to about photographic magnitude 14.5 and many fainter ones.

Luyten has published a separate list of all stars with proper motions in excess of $0 " 5$ and negative declinations (904 stars) and other lists containing 1054 stars with proper motions between $0 " 5$ and $0 " 3$ and declinations south of $-40^{\circ}$, with approximate apparent magnitudes and colours. ${ }^{1}$ 
These lists are essentially complete. Another similar list ${ }^{1}$ of 393 stars within the same limits of proper motions and between declination $0^{\circ}$ and $-40^{\circ}$ is incomplete. Further, a list of 305 stars north of the equator with proper motions between $0 " 2$ and $0 " 5$ has been published by Luyten. ${ }^{2}$

For the northern hemisphere, Ross' lists ${ }^{3}$ are the most extensive ones. They contain stars brighter than the $13^{\text {th }}$ photographic magnitude in about $85 \%$ of this hemisphere (and $20 \%$ of the southern one). Ross lists about 800 stars with proper motions larger than $0 " 3$. Wolf's lists ${ }^{4}$ contain about 1500 stars, mostly brighter than $m=13$, about one-third of which have proper motions exceeding $0 " 3$.

The limit of $0 " 3$ would be a suitable one for the selection of stars for re-measurement. If the still unpublished lists compiled by Luyten would become available the total number of these stars would be about 4000 , about 1500 of them being bright enough for the Astrographic Catalogue plates and many of the remaining ones measurable on the chart plates.

Part of these stars are covered by other independent programmes. For instance, the Ross stars will be measured on $40^{\prime \prime}$ plates taken at the Yerkes Observatory. There is no reason, however, to avoid duplication.

Of special interest will be the improvement of the proper motions of all the white dwarfs known at present. A study of the colours and magnitudes of white dwarfs is being made by Harris at the Yerkes Observatory. Various lists of white dwarfs have been published by Luyten. ${ }^{5}$

\section{Stars in the $O$ associations}

For this programme see Section D (I), pp. 43 and 44 .

\section{(2) PROPER MOTIONS: MERIDIAN OBSERVATIONS}

There are several fundamental problems with regard to galactic structure, which require precise knowledge of proper motions of bright stars $(m<8)$ in a well determined fundamental system. Examples are: the determination of the constants $A$ and $B$ of galactic rotation; the determination of the zero point of the period luminosity curve of Cepheids; the calibration of spectroscopic absolute magnitudes of high luminosity stars. It was decided by the conference that a list of stars for meridian observations be suggested to meridian observers. A first list, containing stars north of declination $-20^{\circ}$, has been made and is added as an Appendix to the present report. ${ }^{6}$ It contains the following stars:

Cepheids (period longer than $\mathrm{I} \cdot 5$ days), brighter than $8 \cdot 0$ visual magnitude in maximum. 
O-B5 stars classified by W. W. Morgan in the revised system of the Yerkes Atlas, brighter than 8.5 visual magnitude.

O-B3 stars according to the Henry Draper Catalogue, still unclassified in the Yerkes system, also brighter than $8 \cdot 5$.

OB super-giants brighter than $8 \cdot 5$ visual magnitude, found in the survey by Nassau and Morgan.

The list contains $106 \mathrm{I}$ stars. Of these, 274 marked ' $\mathrm{P}$ ', may be given priority by those institutions who want to work only on a limited programme; they include all the Cepheids, the super-giants with either a distance modulus below 10.0 or a visual apparent magnitude (corrected for interstellar absorption) brighter than $6 \cdot 0$, and a few additional stars in the nearest associations. For each star are given the BD number and the approximate apparent visual magnitude. Some of the listed stars are among the fundamental stars regularly observed ( $\mathrm{FK}_{3}$ stars, etc.). A few observatories have already indicated that they may carry out at least part of these observations. It is hoped that others will follow so that the highest possible accuracy for these objects can be reached.

For Cepheids, a table of the brighter objects with a list of early epoch references has been published by Parenago in Variable Stars, 6, 102, 1947. Parenago stated that a card catalogue of meridian and astrographic positions of variable stars is kept at the Sternberg Institute; the information on these cards will be made available upon request.

It is intended to publish other lists of stars of particular interest for meridian observers as the need arises. The $\mathrm{M}$ super-giants and the $\mathrm{N}$ and $\mathrm{S}$ stars referred to in Section A (2), may be among these.

\section{(3) PROPER MOTIONS: THE REPETITION OF THE AGK 2}

As was reported by Heckmann, the Hamburg Observatory contemplates repetition of the $\mathrm{AGK}_{2}$ between the years 1956 and 1960 . This large project consists of two parts: (a) the photographic repetition of the $193^{\circ}$ plates and measurement of the relative proper motions; and $(b)$ the meridian observations of reference stars during the same years. Details concerning this project are published elsewhere. ${ }^{1}$ An important question discussed at the conference was, whether the repetition should be made about $1970-80$ as planned originally, or already about 1960 with the consequent reduction in the accuracy of the proper motions obtained from the comparison with AGK 2. The repetition would produce a homogeneous system of 'absolute' proper motions for about 180,000 stars down 
to the I Ith photographic magnitude in the northern hemisphere. Their mean errors would be \pm 0 ".008, for the I 960 repetition.

The importance of this project lies not only in the large number of proper motions of faint stars which will become available for study of various galactic problems, but particularly in the fact that it will furnish the bases for both a more accurate reduction of the old meridian catalogues (magnitude errors!) and for deriving the full profit from the old Astrographic Catalogue positions. Any plate of the Astrographic Catalogue could be re-reduced completely with the aid of improved positions as derived from the new proper motions and present positions. Proper motions derived from the comparison of the old plates with modern ones would have an uncertainty in the reduction to absolute of about \pm 0 "002 only.

For the observation of the reference stars for the repetition of the AGK 2, collaboration with other meridian observatories than Hamburg has already been sought for by Heckmann.

The conference agreed that the project proposed by Heckmann would be one of fundamental importance for future work on the proper motions of faint stars. The idea of repeating the $\mathrm{AGK}_{2}$ in 1960 , instead of in I $970-80$, found strong support; it was generally admitted that the accuracy of the proper motions to be obtained would be quite satisfactory for statistical investigations, and that the special circumstances mentioned by Heckmann (the availability of experienced personnel at Hamburg) fully justify the advanced repetition.

\section{(4) RADIAL VELOGITIES}

The necessity of extensive work on radial velocities has become apparent during the discussion of many topics at the conference; it contrasts strongly with the decreasing interest shown in this type of observational work by some observatories which formerly were active in this field, and it is hoped that the present report will stimulate new activity.

The following summarizes principal programmes, to most of which we have referred already in other sections of this report:

(I) The OB super-giants found in the objective prism surveys, which will give information on the systematic motions at large distances in the galactic plane (Section A (2)).

(2) Faint Cepheids, mainly those of population $I$ in the galactic plane which will serve for the same purpose as the OB super-giants (see Section A (2)). 
(3) RR Lyrae and long-period variables to be found in the surveys described in Section A (I). Such radial velocity programmes will enable us to derive full profit from the surveys of these distant objects. They will provide information on the galactic field of force and on the kinematical differences between discrete period groups among the variables.

(4) Observations of stars in the Kapteyn Selected Areas below $10^{\circ}$ latitude, brighter than I2.5 photographic magnitude, in a region $3.5 \times 3.5$ degrees (see the discussion and the list on pp. 35 and 36 ); in addition to these the high latitude Selected Areas mentioned in Section B (2), and also the test regions at low latitude (see p. 29).

(5) The stars in the $\mathrm{O}$ associations.

(6) The faint main sequence $\mathrm{B}_{2}-\mathrm{B}_{5}$ stars to be used for a fundamental determination of the constant $A$ of Galaxy rotation (Section $\mathrm{D}(2)$ ).

Dr Fehrenbach gave a report on the development of his method of objective prism radial velocity observations. Preliminary work with a $15 \mathrm{~cm}$. refractor has furnished the necessary experience for future work, which will be carried out with a $40 \mathrm{~cm}$. refractor, especially built for this purpose. Its focal length will be $400 \mathrm{~cm}$. and the dispersion of the spectra, I Io A. $/ \mathrm{mm}$. at $\mathrm{H}_{\gamma}$. The magnitude limit will be $\mathrm{I}_{2.5}$ for exposures of 4 hours ( 2 hours for each of the two spectra to be compared with opposite directions of dispersion).

The precision of the radial velocities obtained from one plate will be of the order of $\pm 6 \mathrm{~km}$. $/ \mathrm{sec}$. for the A stars, and somewhat higher, about $\pm 4 \mathrm{~km}$./sec., for the later types. These figures are based on the experience gained with the $15 \mathrm{~cm}$. refractor.

The area measured with the small instrument was $3 \times 4.5$ degrees. A number of plates in low latitude areas has already been taken with this instrument. The measurements will be carried out by the Astrographic Catalogue division of the Paris Observatory.

Fehrenbach's method will prove to be extremely useful for all those programmes where a large number of stars has to be observed in a small area of the sky, like the Selected Areas. These new developments were, therefore, highly appreciated by the conference and it expressed the hope that soon much progress will be made with the measurement of the plates. It therefore adopted the following resolution:

La mesure des vitesses radiales au prisme objectif, telle qu'elle a été mise au point par M. Fehrenbach, est de la plus grande importance pour l'étude du système galactique. La conférence demande instamment que ce travail soit poussé le plus activement possible, à la fois dans l'hémisphère Nord et dans l'hémisphère Sud. 
The method of objective prism radial velocities requires knowledge of the radial velocities of a few standard stars in the field observed. This presents a difficult problem, and requires the co-operation of radial velocity observers equipped with large instruments.

Various participants at the conference (Bok, Lindblad, Melnikov-for Panaeyotov-, Schalén) reported investigation of the method of objective prism radial velocities. Extension of this work with still larger instruments, which perhaps might reach stars of $14^{\text {th }}$ magnitude, would be of the utmost importance, especially for the study of the kinematical properties of the distant parts of our Galaxy. 